\title{
CONFORMIDADE DE ACESSOS E DE SAÍDAS DE POSTOS DE OPERAÇÃO EM TRATORES AGRÍCOLAS SEGUNDO NORMA NBR/ISO 4252
}

\author{
DANIELLE M. P. MATTAR ${ }^{1}$, ARNO U. DALLMEYER ${ }^{2}$, JOSÉ F. SCHLOSSER ${ }^{3}$, \\ MARÇAL E. DORNELLES ${ }^{4}$
}

\begin{abstract}
RESUMO: Este trabalho teve como objetivo avaliar a conformidade dos acessos e de saídas dos postos de operação em tratores agrícolas, por meio de população amostral dos tratores fabricados no Brasil. Para a avaliação, diversos itens referentes aos acessos e às saídas dos postos de operação foram analisados qualitativa e quantitativamente com base nos padrões da Norma NBR/ISO 4252 . Ao final do trabalho, foi observado que os tratores classificados na Classe III, quanto à potência nominal do motor, atendem ao maior número de requisitos da Norma em comparação a tratores da Classe II. Dentre os itens avaliados, a altura da plataforma até o teto dos postos de operação foi atendida por todos os modelos. As presenças de batentes de proteção sobre os degraus e de corrimão para o operador foram os itens em maior desconformidade sobre os tratores. Como resultado médio, a população amostral foi conforme em $66 \%$ dos itens analisados. Os tratores dotados de motores de maior potência apresentaram, em geral, acessos e saídas dos postos de operação mais ergonômicos e seguros. De maneira geral, conclui-se pela baixa conformidade dos itens de ergonomia e de segurança dispostos ao operador sobre os postos de operação em tratores agrícolas nacionais.
\end{abstract}

PALAVRAS-CHAVE: ergonomia, máquinas agrícolas, segurança operacional.

\section{CONFORMITY OF THE ACCESSES AND EXITS OF AGRICULTURAL TRACTORS WORKPLACES ACCORDING TO NBR/ISO 4252 STANDARD}

\begin{abstract}
This work had as objective to evaluate the conformity of agricultural tractors workplaces accesses and exits made in Brazil. For this evaluation, the workplaces were analyzed according to NBR/ISO 4252 Standard. It was observed that the tractors classified in Class III, in relation to power engine, was better then tractors in Class II. Among the parameters evaluated, the platform weight of operator workplace was attended by all the tractors. The items in less conformoty in agricultural tractors are the doorpost of protection on the steps and the handrail to the operator. As results, the sampling population was conformed in $66 \%$ of the analyzed items. The tractors with more powerful engines presented workplaces accesses and exits more ergonomic and safety. Thus, it was concluded by the low conformity of operators workplace in the Brazilian agricultural tractors the minimum parameters of ergonomic and safety for the operator.
\end{abstract}

KEYWORDS: ergonomics, agricultural machines, operational safety.

\section{INTRODUÇÃO}

A agricultura brasileira, nos moldes atuais, está diretamente ligada ao uso intensivo de máquinas e implementos agrícolas. A possibilidade de expansão de áreas agrícolas e a necessidade de aumento na produção de alimentos levam produtores rurais a adquirirem máquinas agrícolas, aquisição que ocorre pela necessidade de diminuição de esforços físicos necessários para a execução de tarefas, visando à maior eficiência e à diminuição de custos. Todavia, a maior utilização de máquinas aumenta a exposição dos trabalhadores a riscos de acidentes laborais.

\footnotetext{
${ }^{1}$ Eng ${ }^{a}$ Agrícola, Mestranda em Engenharia Agrícola, Centro de Ciências Rurais, Programa de Pós-Graduação em Engenharia Agrícola, UFSM, Santa Maria - RS, Fone: (0xx55) 8135.8995, danielle_mattar@yahoo.com.br

${ }^{2}$ Eng ${ }^{\mathrm{o}}$ Agrônomo, Professor Titular, Centro Tecnológico, UFSM, Santa Maria - RS.

${ }^{3}$ Eng ${ }^{0}$ Agrônomo, Professor Titular, Departamento de Engenharia Rural, UFSM, Santa Maria - RS.

${ }^{4}$ Eng ${ }^{0}$ Agrônomo, mestrando em Engenharia Agrícola. UFSM, Santa Maria - RS.

Recebido pelo Conselho Editorial em: 6-4-2008
}

Aprovado pelo Conselho Editorial em: 27-9-2009

Eng. Agríc., Jaboticabal, v.30, n.1, p.74-81, jan./fev. 2010 
A operação segura envolve fatores de ergonomia e de segurança, tanto na fabricação, quanto no uso das máquinas.

Conforme estimativa de FRANCHINI (2007) e ANFAVEA (2007), há mais de 450 mil tratores em uso no Brasil, sendo de fundamental importância que essas máquinas possuam bons acessos e saídas aos postos de operação, como ação preventiva de acidentes para os operadores agrícolas.

Dentre as máquinas introduzidas no meio rural, o trator agrícola destaca-se principalmente pela aplicabilidade, como fonte de potência e de tração em imensa gama de tarefas dentro da propriedade agrícola (ROZIN, 2004). Com a modernização da agricultura, é visível a melhoria sobre os postos de operação dos tratores agrícolas nacionais com aplicação de técnicas ergonômicas (FRANCHINI, 2007), porém muitas exigências ainda são negligenciadas.

O projeto de máquinas agrícolas com enfoque ergonômico contribui para melhorar a eficiência, a confiabilidade e a qualidade operacional, buscando o aperfeiçoamento da relação homem/máquina. Nos países da União Europeia, há obrigatoriedade de que os fabricantes de máquinas agrícolas ou florestais obedeçam às diretivas de segurança (ALONÇO, 2004), onde as empresas se responsabilizam por eventuais erros projetuais ou danos de integridade física sobre os operadores. Um processo consolidado de desenvolvimento de novas máquinas deve atender às necessidades de segurança e de ergonomia (ROMANO, 2003). A ergonomia de concepção do posto de operação deve fazer parte do projeto das máquinas agrícolas (SANTOS, 1999).

Conforme ALONÇO et al. (2006), é admitido que o perigo seja inerente aos sistemas técnicos, porém, na fase de projeto do produto, todas as barreiras e salvaguardas necessárias devem ser implementadas de modo a permitir que não haja perigos potenciais geradores de acidentes envolvendo o operador. Segundo CORRÊA (2007), ainda não há, no Brasil, incorporação satisfatória de dispositivos de segurança nas máquinas agrícolas industrializadas.

Dentre os principais acidentes e lesões sobre os operadores de tratores agrícolas estão aqueles ligados aos acessos e às saídas dos postos de operação. Para YADAV \& TEWARI (1998), o melhor posto de operação projetado para tratores agrícolas será aquele em que o operador irá cometer menor número de erros durante as tarefas de maior dificuldade, utilizando menor energia metabolizada. Devido ao esforço necessário da estrutura corporal para ter acesso ou deixar a plataforma de operação de tratores, fazem-se necessários constantes estudos e aperfeiçoamentos de acessos e saídas dos postos de operação das máquinas agrícolas. Um trator agrícola ergonomicamente mal projetado aumenta o nível de estresse físico e mental sobre o operador, levando-o a cometer erros (atos inseguros) que podem resultar em acidentes (WITNEY, 1988; MÁRQUEZ, 1990; YADAV \& TEWARI, 1998).

Conforme COUTO (2008), em torno de $20 \%$ dos acidentes de trabalho na agricultura são relativos a tarefas com tratores agrícolas, sendo 54\% de acidentes leves e 14,8\% dos acidentes graves causados devido à condição de equipamentos inadequados, ou seja, ocorrem naquelas condições em que itens de segurança não estão adequados; onde a falta de piso antiderrapante sobre os degraus de acesso de um trator agrícola, por exemplo, poderá levar a lesões corporais do operador.

No Brasil, a padronização dos projetos referentes aos tratores fabricados nacionalmente está assegurada pela Norma NBR 4252 (1999); mesmo assim, observa-se grande disparidade entre as medidas relativas aos acessos e às saídas dos postos de operação dos tratores. O correto dimensionamento e a adequação de acessos e saídas dos postos de operação dos tratores, conforme as medidas corporais dos futuros operadores, contribuem para que se tenha menor exposição a riscos e, com isso, menor número de acidentes de trabalho. Dentre os aspectos de segurança e de ergonomia na atividade de operação dos tratores agrícolas, a questão de acesso e de dimensões dos postos de operação está entre os que assumem maior importância.

O objetivo deste trabalho foi levantar informações a respeito dos acessos e das saídas dos postos de operação de diferentes tratores agrícolas fabricados nacionalmente e realizar avaliação de conformidade com a Norma NBR 4252. 


\section{MATERIAL E MÉTODOS}

Para que o levantamento a respeito das medidas das partes dos acessos e das saídas dos postos de operação dos tratores agrícolas de rodas fosse realizado, o grupo de trabalho visitou revendas e propriedades agrícolas onde os tratores amostrados foram medidos e analisados.

Pela dificuldade em se conseguir analisar todos os modelos nacionais, a seleção dos modelos amostrais foi definida de maneira a abranger um número limitado de tratores de rodas para cada classe de potência, conforme classificação da ANFAVEA (2007) (Tabela 1).

TABELA 1. Classes de potência nominal do motor dos tratores agrícolas. Classes of the engine power of Agricultural tractors.

\begin{tabular}{ccc}
\hline Classes & Faixa de Potência $(\mathrm{kW})$ \\
\hline I & $<36$ \\
II & $36-73$ \\
III & $74-147$ \\
IV & Acima de 147 \\
\hline
\end{tabular}

Fonte: Adaptado a partir de ANFAVEA (2007).

Tratores de rodas com potência nominal do motor inferior a $36 \mathrm{~kW}$ e aqueles com potência maior do que $147 \mathrm{~kW}$ não foram inclusos para a seleção devido à baixa participação de vendas no mercado nacional. Dessa maneira, todos os modelos nacionais comercializados e classificados como classes de potência II ou III e com bitola superior a $1.150 \mathrm{~mm}$ concorreram à seleção. A seleção do número de modelos de cada marca comercial fabricada e comercializada nacionalmente deu-se em função da participação das cinco principais marcas sobre as vendas totais realizadas de janeiro a novembro de 2007 (ANFAVEA, 2007). Posteriormente, dentro da gama de modelos de cada marca, foram escolhidos aqueles modelos que apresentaram as maiores vendas, escolhidos em ordem decrescente, até que o número mínimo de modelos a serem analisados, estimado estatisticamente, fosse atendido. A participação relativa de cada marca na venda total de tratores foi considerada na eleição do número total de modelos a ser analisado. Na Tabela 2, descrevem-se os modelos de tratores agrícolas analisados.

TABELA 2. Tratores de rodas analisados conforme Norma NBR 4252. Wheel tractors analyzed according to the NBR/ISO 4252 Standard.

\begin{tabular}{cccc}
\hline Marca & \multicolumn{2}{c}{ Classe de Potência } & Total \\
\cline { 2 - 4 } Comercial & II & III & 1 \\
\hline Case & - & MX 180 & 3 \\
\hline John Deere & $5605 / 4,5705 / 4$ & $6415 / 4$ & 8 \\
\hline Massey Ferguson & $283 / 4,292 / 4,275 / 4,275,275 E / 4,250 / 4$ & $7630 / 4$, TM 150/4 & 4 \\
\hline New Holland & TL 75E/4, TL 85E/4 & BH 180/4, BM 110/4, BM 100/4 & 5 \\
\hline Valtra & $785 / 4$, BL 88/4 & 9 & 21 \\
\hline TOTAL & 12 & & \\
\hline
\end{tabular}

Todos os tratores agrícolas analisados por esta pesquisa possuíam até, no máximo, 6 meses de uso, ou máximo de 200 horas de uso; se o trator em questão ultrapassasse qualquer um desses requisitos, foi desconsiderado e buscou-se por novo trator de mesmo modelo para que não houvesse desgaste do trator que pudesse mascarar informações de projeto da máquina.

Foram utilizados diversos equipamentos para a coleta dos dados ergonométricos sobre os tratores agrícolas, tais como: trena; transferidor; máquina fotográfica, pranchetas e ficha de anotações. Um compasso de madeira, utilizado para a determinação do ângulo de abertura da porta de acesso ao posto de operação de tratores cabinados, foi desenvolvido, conforme as especificações descritas pela norma ISO 4252 e pela sua versão brasileira, em homologação, NBR/ISO 4252. 
Partes dessas especificações estão normalizadas em nível internacional, podendo servir de orientação para o aperfeiçoamento das máquinas nacionais.

Na Figura 1, estão descritas algumas das especificações presentes na Norma NBR/ISO 4252 (ABNT, 1999) e os respectivos valores-padrão a serem seguidos pelos fabricantes (Tabela 3) e que foram considerados para a avaliação deste trabalho.

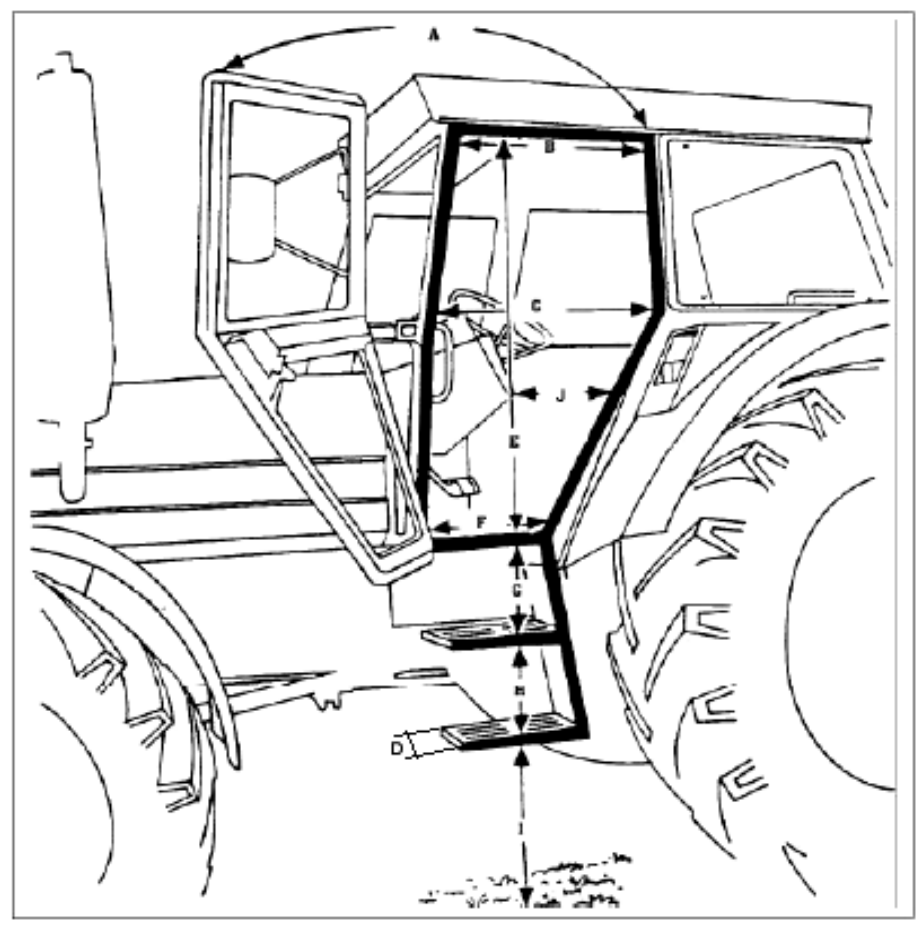

FIGURA 1. Identificação de itens analisados sobre o posto de operação dos tratores agrícolas. Identification items about the workplace accesses and exits in agricultural tractors.

TABELA 3. Dimensões estabelecidas pela norma NBR 4252 de acessos e saídas dos postos de operação. Measurements observed according to the NBR 4252 Standard.

\begin{tabular}{clr}
\hline Itens & \multicolumn{1}{c}{ Dispositivos de Acesso } & Medidas \\
\hline A & Ângulo de abertura da porta (valor mínimo) & $120^{\circ}$ \\
B & Largura superior da porta (valor mínimo) & $670 \mathrm{~mm}$ \\
C & Maior largura da porta (valor mínimo) & $750 \mathrm{~mm}$ \\
D & Profundidade do degrau (valor mínimo) & $150 \mathrm{~mm}$ \\
E & Altura da porta do trator ou da plataforma ao toldo (valor mínimo) & $1.330 \mathrm{~mm}$ \\
F & Largura do degrau e a entrada da plataforma (valor mínimo) & $270 \mathrm{~mm}$ \\
G & Distanciamento do degrau à plataforma (valor máximo) & $300 \mathrm{~mm}$ \\
H & Distanciamento de um degrau ao outro sucessivo (valor máximo) & $300 \mathrm{~mm}$ \\
I & Altura do primeiro degrau em relação ao solo (valor máximo) & $550 \mathrm{~mm}$ \\
J & Distância da entrada ao primeiro obstáculo (valor mínimo) & $450 \mathrm{~mm}$ \\
\hline
\end{tabular}

\section{RESULTADOS E DISCUSSÃO}

Nas Tabelas 4 e 5, apresentam-se os itens analisados e as medidas tomadas nos tratores agrícolas, que descrevem as medidas importantes para a análise de segurança e de ergonomia, conforme citado na Figura 1. 
TABELA 4. Dimensões observadas (mm) nos tratores analisados. Measurements observed (mm) of the analyzed tractors.

\begin{tabular}{|c|c|c|c|c|c|c|c|}
\hline $\begin{array}{c}\text { Marca } \\
\text { Comercial }\end{array}$ & Modelo & $\begin{array}{l}\text { Potência Nominal } \\
\qquad(\mathrm{kW})\end{array}$ & $\begin{array}{l}\text { Distância do } 1^{\underline{0}} \\
\text { Degrau ao Solo }\end{array}$ & $\begin{array}{c}\text { Distância do } \\
1^{\circ} \text { ao } \\
2^{\underline{o}} \text { Degrau }\end{array}$ & $\begin{array}{c}\text { Distância do } \\
2^{\underline{0}} \text { ao } \\
3^{0} \text { Degrau }\end{array}$ & $\begin{array}{l}\text { Largura } \\
\text { do } \\
\text { Degrau }\end{array}$ & $\begin{array}{c}\text { Altura do } \\
\text { Degrau }\end{array}$ \\
\hline \multirow{6}{*}{$\begin{array}{l}\text { Valtra } \\
\text { (VA) }\end{array}$} & 110 & 81 & 640 & 190 & - & 360 & 40 \\
\hline & BL 88 & 64,8 & 400 & 350 & - & 250 & 3 \\
\hline & 785 & 55,2 & 600 & 210 & - & 420 & 40 \\
\hline & 685 & 45,7 & 490 & 195 & - & 340 & 40 \\
\hline & BM 125i & 92,1 & 600 & 210 & 210 & 330 & 40 \\
\hline & BH 180 & 132,6 & 550 & 360 & 250 & 460 & 40 \\
\hline \multirow{5}{*}{$\begin{array}{l}\text { New } \\
\text { Holland } \\
(\mathrm{NH})\end{array}$} & $85 \mathrm{E}$ & 66,3 & 540 & 255 & 285 & 370 & 35 \\
\hline & $75 \mathrm{E}$ & 57,5 & 537 & 255 & 286 & 355 & 35 \\
\hline & 7630 & 77,3 & 535 & 240 & 220 & 255 & 35 \\
\hline & TT 55 & 40,5 & 540 & 200 & - & 200 & 20 \\
\hline & TM 135E & 99,4 & 510 & 220 & 220 & 360 & 35 \\
\hline \multirow{3}{*}{$\begin{array}{l}\text { John Deere } \\
\text { (JD) }\end{array}$} & $5605 / 4$ & 55,2 & 550 & 300 & - & 300 & 25 \\
\hline & $5705 / 4$ & 62,6 & 640 & 350 & - & 330 & 25 \\
\hline & $6415 / 4$ & 78,1 & 560 & 300 & 280 & 330 & 30 \\
\hline \multirow{6}{*}{$\begin{array}{l}\text { Massey } \\
\text { Ferguson } \\
\text { (MF) }\end{array}$} & $680 / 4$ & 127,4 & 640 & 280 & 295 & 320 & 40 \\
\hline & $250 / 4$ & 36,8 & 480 & 230 & - & 190 & 5 \\
\hline & $292 / 4$ & 77,3 & 410 & 240 & 250 & 335 & 25 \\
\hline & $275 / 4$ & 55,2 & 500 & 350 & - & 355 & 35 \\
\hline & $275 / 2$ & 55,2 & 600 & 270 & - & 210 & 35 \\
\hline & $283 / 4$ & 63,3 & 510 & 360 & - & 370 & 38 \\
\hline Case & MX 180/4 & 132,6 & 510 & 220 & 260 & 370 & 40 \\
\hline \multicolumn{2}{|c|}{ Média } & 77,9 & 540,10 & 265,95 & 255,60 & 324,29 & 31,48 \\
\hline
\end{tabular}

TABELA 5. Dimensões observadas (mm) nos tratores analisados. Measurements observed (mm) of the analyzed tractors.

\begin{tabular}{|c|c|c|c|c|c|c|}
\hline $\begin{array}{c}\text { Marca } \\
\text { Comercial }\end{array}$ & Modelo & $\begin{array}{c}\text { Profundidade do } \\
1^{\circ} \text { Degrau } \\
\end{array}$ & $\begin{array}{c}\text { Profundidade do } \\
2^{\circ} \text { Degrau }\end{array}$ & $\begin{array}{l}\text { Largura da } \\
\text { Plataforma }\end{array}$ & $\begin{array}{c}\text { Altura da } \\
\text { Plataforma }\end{array}$ & $\begin{array}{c}\text { Distância ao } 1^{0} \\
\text { Obstáculo }\end{array}$ \\
\hline \multirow{6}{*}{$\begin{array}{l}\text { Valtra } \\
\text { (VA) }\end{array}$} & 110 & 50 & - & 230 & 1.500 & 600 \\
\hline & BL 88 & 45 & - & 520 & 1.550 & 430 \\
\hline & 785 & 150 & - & 735 & 1.580 & 350 \\
\hline & 685 & 150 & - & 210 & 1.600 & 310 \\
\hline & BM 125i & 100 & 100 & 330 & 1.380 & 570 \\
\hline & BH 180 & 180 & 480 & 340 & 1.420 & 610 \\
\hline \multirow{5}{*}{$\begin{array}{l}\text { New Holland } \\
\text { (NH) }\end{array}$} & $85 \mathrm{E}$ & 200 & 200 & 400 & 1.600 & 560 \\
\hline & $75 \mathrm{E}$ & 200 & 200 & 410 & 1.580 & 430 \\
\hline & 7630 & 140 & 140 & 700 & 1.400 & 355 \\
\hline & TT 55 & 130 & - & 420 & 1.505 & 360 \\
\hline & TM 135E & 200 & 200 & 410 & 1.420 & 600 \\
\hline \multirow{3}{*}{$\begin{array}{l}\text { John Deere } \\
\text { (JD) }\end{array}$} & $5605 / 4$ & 190 & - & 430 & 1.460 & 480 \\
\hline & $5705 / 4$ & 150 & - & 430 & 1.470 & 580 \\
\hline & $6415 / 4$ & 190 & 190 & 650 & 1.390 & 650 \\
\hline \multirow{6}{*}{$\begin{array}{l}\text { Massey } \\
\text { Ferguson } \\
\text { (MF) }\end{array}$} & $680 / 4$ & 160 & 160 & 480 & 1.600 & 650 \\
\hline & $250 / 4$ & 30 & - & 330 & 1.590 & 460 \\
\hline & $292 / 4$ & 100 & 100 & 240 & 1.580 & 430 \\
\hline & $275 / 4$ & 150 & - & 255 & 1.590 & 390 \\
\hline & $275 / 2$ & 50 & - & 240 & 1.590 & 425 \\
\hline & $283 / 4$ & 155 & - & 250 & 1.580 & 390 \\
\hline Case & MX 180/4 & 200 & 200 & 400 & 1.400 & 580 \\
\hline \multicolumn{2}{|c|}{ Média } & 139,05 & 197,00 & 435,71 & $1.478,33$ & 486,19 \\
\hline
\end{tabular}


Os tratores analisados apresentaram conformidade média em $66 \%$ dos itens analisados por esta pesquisa. Na Figura 2, apresentam-se os percentuais de atendimento de conformidade para cada um dos itens analisados.

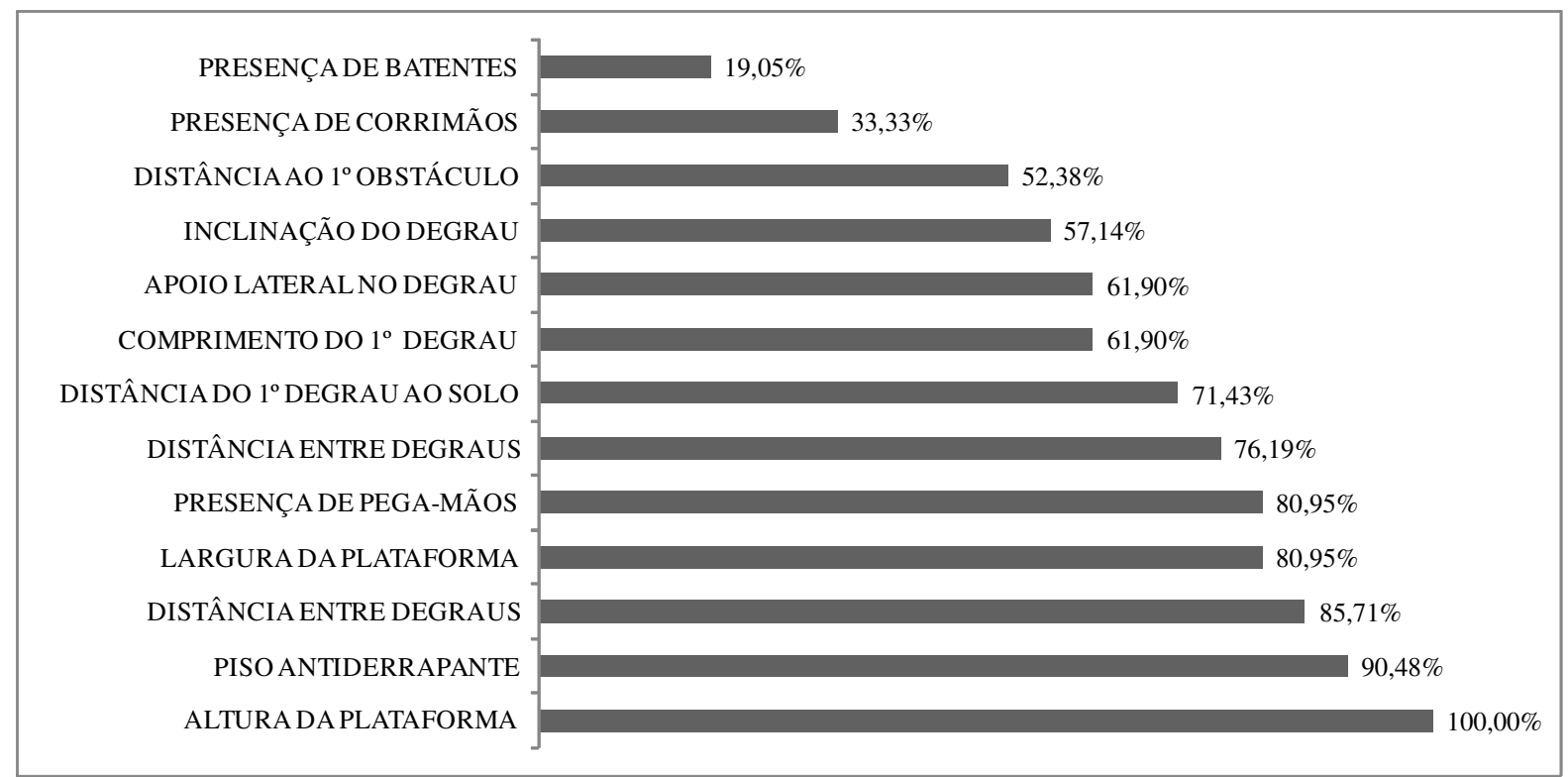

FIGURA 2. Conformidade dos tratores quanto aos acessos e às saídas aos postos de operação de acordo com as exigências mínimas da Norma NBR/ISO 4252. Conformity of the tractors about the workplace accesses and exits according to the NBR/ISO 4252 Standard.

Dentre os itens, houve destaque quanto aos valores de altura de plataforma, sendo atendida por todos os modelos. Em segundo e terceiro lugares, os itens presença de piso antiderrapante e distância mínima entre degraus foram atendidos por 90,48 e 85,71\% dos modelos, respectivamente. Durante o acesso à máquina agrícola, o operador impõe grande esforço sobre os pontos de contato dos pés com os degraus; nesse sentido, são exigidas estruturas antiderrapantes como proteção a possíveis escorregões e tombos em altura elevada, a fim de evitar lesões corporais.

Dentre os itens em maior desconformidade, a presença de batentes sobre os degraus e a presença de corrimãos tiveram destaque, sendo atendidas por apenas $19,05 \%$ e 33,33\% dos tratores, respectivamente. Segundo ROBIN (1987), colaborando com a Norma NBR/ISO 4252, faz-se necessária a colocação de corrimãos em ambos os lados das vias de acesso, servindo de apoio aos tratoristas, tanto para acessar como para deixar o posto de operação. $\mathrm{O}$ mesmo autor cita que a disposição dos degraus seja de forma inclinada em relação à vertical para facilitar o acesso. Neste trabalho, foi observado que apenas $57,14 \%$ dos modelos apresentavam degraus inclinados.

Outro item que chamou a atenção, devido ao baixo atendimento de conformidade na população amostral, foi a distância máxima do primeiro degrau ao solo $(71,43 \%)$. A disposição do primeiro degrau à linha do solo faz com que, em muitos casos, os operadores tenham de realizar grandes esforços em posição inoportuna e com grande impacto negativo sobre os joelhos para ter acesso aos postos de operação. Conforme SCHLOSSER (2007), em pesquisa realizada com operadores no Rio Grande do Sul, a maioria dos operadores jovens costuma jogar-se diretamente da plataforma do posto de operação ao solo por considerar a estrutura de saída de alguns postos de operação inadequados ou inseguros.

Um detalhe importante observado nesta pesquisa é que os tratores de classe III, quanto à potência nominal do motor (ANFAVEA, 2007), apresentaram aprovação de 73,08\%, enquanto os tratores de classe II tiveram aprovação de $61,54 \%$. De maneira geral, isso pode ser explicado 
levando-se em conta que os tratores com motores de maior potência nominal possuem maior valor comercial. Assim, a adição de itens de ergonomia, segurança e conforto mais adequados, e de maior valor sobre o posto de operação do trator, impactam relativamente menos sobre o preço final. Essa tendência é mais bem visualizada na Figura 3, em que são apresentados os resultados de conformidade para cada modelo pesquisado na ordem crescente quanto à potência nominal do motor, para as diferentes marcas analisadas.

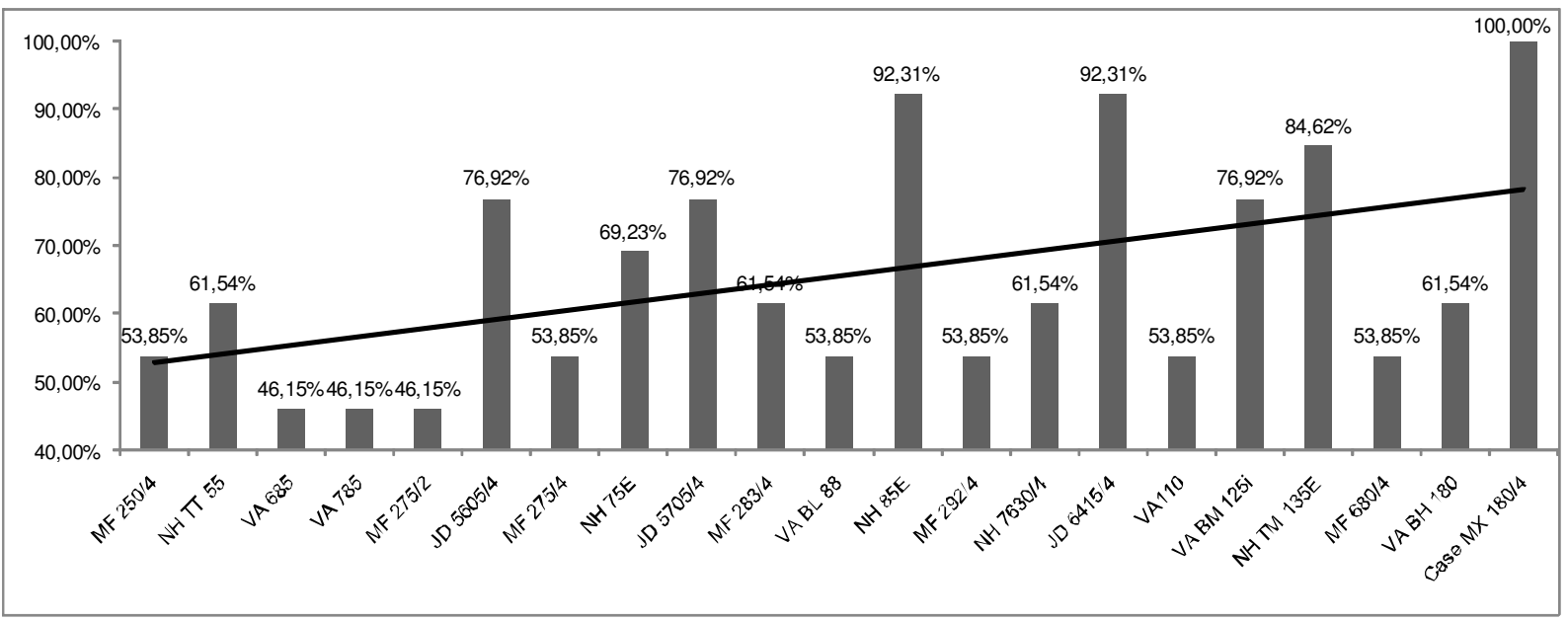

FIGURA 3. Tendência de atendimento à Norma NBR 4252, conforme aumento da potência nominal do motor sobre os tratores agrícolas. Service trend according to the 4253 Standard, about the engine power increase of agricultural tractors.

Infelizmente, para o Brasil, já foi constatado por meio de outros trabalhos (DEBIASI et al., 2004) que itens de conforto e de segurança não são prioridade na hora de aquisição de tratores agrícolas, sendo que o preço final ganha destaque na decisão do consumidor brasileiro. Assim, os fabricantes nacionais seguem a tendência do consumidor, priorizando a obtenção de menor preço final, deixando de lado, muitas vezes, a observação de itens de ergonomia e de segurança fundamentais ao bom desempenho e segurança do operador.

\section{CONCLUSÕES}

Os tratores nacionais analisados não atenderam satisfatoriamente às exigências mínimas da Norma NBR 4252 quanto ao acesso e à saída dos postos de operação.

Foi determinada tendência à conformidade dos acessos e saídas dos postos de operação com o aumento da potência nominal do motor dos tratores agrícolas analisados.

Assim, foi determinado com este trabalho a necessidade de maior atenção por parte dos fabricantes nacionais de tratores agrícolas à importância da adequação às exigências da Norma NBR 4252. Portanto, estando em conformidade com a norma, são atingidas melhores condições ergonômicas aos operadores, como ação preventiva a possíveis lesões corporais e acidentes laborais, resultantes da relação de trabalho com tratores agrícolas.

\section{REFERÊNCIAS}

ALONÇO, A. dos S. Metodologia de projeto para segurança em máquinas agrícolas. 2004. 208f. Tese (Doutorado em Engenharia Mecânica) - Universidade Federal de Santa Catarina, Florianópolis, 2004.

ALONÇO, A. dos S.; MEDEIROS, C.A.; MEDEIROS, F.A.; WERNER, V. Análise ergonômica do trabalho em atividades desenvolvidas com uma roçadora manual motorizada. Ciência Rural, Santa Maria, v.36, n.5, set./out., 2006. 
ANFAVEA. ASSOCIAÇÃO NACIONAL DOS FABRICANTES DE VEÍCULOS

AUTOMOTORES. Anuário estatístico da indústria automotiva brasileira. Disponível em: <http://www.anfavea.com.br>. Acesso em: 10 out. 2007.

ASSOCIAÇÃO BRASILEIRA DE NORMAS TÉCNICAS. Tratores agrícolas: acomodação do assento do operador - dimensões: NBR/ISO 4252. Rio de Janeiro, 1999.

CORRÊA, I. M. Pequeno, mas seguro. Revista Cultivar Máquinas, Pelotas, n.62, p.14-16, 2007.

COUTO, J.L.V. Riscos de acidentes com tratores agrícolas. Rio de Janeiro: Universidade Federal do Rio de Janeiro. Disponível em: <http://www.segurancaetrabalho.com.br/download/tratorescouto.doc>. Acesso em: 10 dez. 2008.

DEBIASI, H.; SCHLOSSER, J.F.; PINHEIRO, E.D. Características ergonômicas dos tratores agrícolas utilizados na região central do Rio Grande do Sul. Ciência Rural, Santa Maria v.34, n.6, p.1.807-1.811, 2004.

FRANCHINI, D. Análise do nível de vibrações verticais no assento de um trator agrícola. 2007. 139 f. Dissertação (Mestrado) - Universidade Federal de Santa Maria, Santa Maria, 2007.

MÁRQUEZ, L. Solo tractor'90. Madrid: Laboreo, 1990. 231 p.

ROBIN, P. Segurança e ergonomia em maquinaria agrícola: tratores agrícolas. São Paulo: IPT, 1987. $26 \mathrm{p}$.

ROMANO, L. N. Modelo de referência para o processo de desenvolvimento de máquinas agrícolas. 2003. 321 f. Tese (Doutorado em Engenharia Mecânica) - Universidade Federal de Santa Catarina, Florianópolis, 2003.

ROZIN, D. Conformidade do posto de operação de tratores agrícolas nacionais com Normas de ergonomia e segurança. 2004. 204 f. Dissertação (Mestrado) - Universidade Federal de Santa Maria, Santa Maria, 2004.

SANTOS, C.M.D. Móveis ergonômicos. Revista Proteção, São Paulo, p.62-65, set. 1999.

SCHLOSSER, J.F. Acesso e saída dos postos de operação: material didático. Santa Maria: Universidade Federal de Santa Maria, 2007. 21 p. CD-ROM.

WITNEY, B. Choosing and using farm machines. Harlow: Longman Scientific and Technical, 1988. p.28-94.

YADAV, R.; TEWARI, V.K. Tractor operator seat workplace design: a review. Journal of Terramechanics, Silsoe, p.41-53, 1998. 